\title{
COMPARATIVE EVALUATION OF FADING CHANNEL MODEL SELECTION FOR MOBILE WIRELESS TRANSMISSION SYSTEM
}

\author{
Z.K. Adeyemo ${ }^{1}$ D.O. Akande, F.K. Ojo and H.O. Raji \\ Department of Electronic \& Electrical Engineering, Ladoke Akintola University of \\ Technology, Ogbomoso, Nigeria. \\ zkadeyemo@yahoo.com, akandedamilare@yahoo.com, \\ festusoluseye@yahoo.com, hammyrajegmail.com \\ ${ }^{1}$ Author to whom correspondence should be sent
}

\begin{abstract}
This paper compares the performance of Rayleigh, Rician and Log-normal distributions in mobile wireless transmission system. In these distributions link quality cannot be predicted accurately enough to achieve reliable performance because of the unpredictable nature of the time-varying channel at different mobile speeds in sub-urban environment and as a result it will be very difficult to select the appropriate model in the system design for proper implementation. Binary Phase Shift Keying signaling scheme is used to modulate the data to be analyzed for efficient transmission over the three different distributions at mobile speeds of $30 \mathrm{~km} / \mathrm{h}, 60 \mathrm{~km} / \mathrm{h}$ and $90 \mathrm{~km} / \mathrm{h}$ at carrier frequency of $900 \mathrm{MHz}$. Square root raised cosine filter is used to convert the modulated signal to analog signal suitable for transmission. The received bits through the channel without mitigating technique over the three scenarios are analyzed and Bit Error Rate (BER) computed for comparison. The results obtained showed that Rayleigh distribution has the highest BER, while Rician distribution has the BER between the Rayleigh and log-normal at the different speeds considered. This implies that Rayleigh distribution is the best model to be adopted by communication systems engineers.
\end{abstract}

\section{KEYWORDS}

Multipath, BPSK, Bit Error rate, Rayleigh, Rician, Log-normal

\section{INTRODUCTION}

Wireless communication is one of the most vibrant areas in the communication field today due to a confluence of several factors. In telecommunication, wireless communication may be used to transfer information over short and long distances. It encompasses various types of systems like fixed and mobile portable two-way radios, cellular telephones, personal digital assistants (PDAs), and wireless networking [1]. Wireless operations permits services, such as long range communications, that are impossible or impractical to implement with the use of wires. The transmission services offered by wireless communication can be categorized into fixed wireless transmission and Mobile wireless transmission. In fixed wireless transmission, the transmitter and the receiver are static while mobile wireless transmission, the transmitter and the receiver are in motion but are moving at different speeds $[2,3,4,5]$.

Channel is the physical medium that is used to send the signal from the transmitter to the receiver [6]. More recently, it is also used to describe a radio terminal that is attached to a high speed mobile platform. According to [6], there are several kinds of communication impairments that are typical of the mobile wireless environment. Impairments may result mainly from multipath transmission, attenuation of signal power from large objects, relative transmitterreceiver motion, interference, spreading of electromagnetic power and thermal or background DOI : 10.5121/ijwmn.2012.4610 
International Journal of Wireless \& Mobile Networks (IJWMN) Vol. 4, No. 6, December 2012

noise. Multipath propagation in connection with the movement of the receiver and/or the transmitter leads to drastic and random fluctuations of the received signal thus resulting into a phenomenon referred to as fading. In mobile radio communication, the emitted electromagnetic waves often do not reach the receiving antenna directly due to obstacles blocking the line-ofsight path and causes reflection, diffraction, and scattering. In fact, the received signals are a superposition of waves coming from all directions. The radio spectrum available for wireless communications is extremely scarce, while demand for mobile and personal communication is growing at a rapid space $[4,7,8,9,10]$. The wireless channel poses a severe challenge as a medium for reliable high-speed communication. It is not only susceptible to noise, interference and other channel impediments, but nature of these impediments change over time in unpredictable ways due to user movements causing the received signal to fluctuate or vary [3]. These defining characteristics of the mobile wireless channel can be interpreted as variations of the channel strength over time and frequency. The major effect of multipath propagation is Doppler spread which has a negative influence on the transmission characteristics of the mobile radio channel. This effect causes a frequency shift of each of the partial waves [11,12]. One of the most common methods for characterizing a fading channel is the use of a probability density function (pdf), which represents the probability density of the received signal strength. The shape of a pdf determines the performance of a wireless receiver in the presence of noise and interference [7,6]. Proper characterization of fading pdfs also impacts the design and use of mitigating techniques for a communication link [3]. The received envelope is very important because it determines the range of received signal strengths. The envelope of the received signal can determine the ultimate Shannon channel capacity of a fading wireless link since received power is proportional to the square of received envelope [13].

Most parts of communication systems over time-varying channels are designed to achieve required performance under the worst-case channel conditions. Large margins are taken into account at the designed stage. A major problem is that such margins do not allow for taking maximum advantage on the available channel capacity especially in the case of varying channels $[14,15,16,17,18,19,20]$. Nowadays, the communication systems engineer has at their finger tips many distributions to predict the behaviour of radio communication systems over fading channel. Many researchers have worked on some of the appropriate model for different environments in fixed wireless communication. But to the best of authors' knowledge, up to now much work has not been carried out on the model selection at different mobile speeds in sub urban environment. The models/distribution to be evaluated are Rayleigh, Rician and Lognormal distributions, hoping that the one with the highest BER will be the best model to choice in the design of communication system before the implementation. The generated data is converted to bits, reshaped and modulated with BPSK signaling scheme. Square root raised cosine filter of a roll-off factor of 0.25 is used to reduce the spectral occupancy and converts the BPSK modulated signal to analog signal suitable for transmission over the time-varying channel. The filter BPSK modulated signals are transmitted through Rayleigh, Rician and lognormal fading distributions. The received signal envelope is demodulated by BPSK demodulator, filtered again to obtain the faded bits. Then, BER which is used as a figure of merit for the evaluation of each of the three scenarios is obtained for comparison.

The results obtained showed that Rayleigh distribution has the highest BER at all the signal to noise ratio, while Rician distribution has its BER values between the Rayleigh and log-normal at mobile speeds of $30 \mathrm{~km} / \mathrm{h}, 60 \mathrm{~km} / \mathrm{h}$ and $90 \mathrm{~km} / \mathrm{h}$ when measure at the same distance. This implies that Rayleigh fading channel is the best model to be adopted by communication systems engineers because if the system engineer can design a robust system in this environment, it will be better in other environments. 
International Journal of Wireless \& Mobile Networks (IJWMN) Vol. 4, No. 6, December 2012

\section{SYSTEM MODEL}

In this paper, the system model consists of the transmitter, the three different fading scenarios and the receiver. This is shown in Figure 1. The transmitter is the sub-system that takes the information signal and processes the randomly generated data prior to transmission.

The received signal, $r(t)$, is given by:

$$
r(t)=m(t) * s(t)
$$

where $m(t)$ is the fading distribution component

$\mathrm{s}(\mathrm{t})$ is the BPSK signal

Note that: the fading distribution component $m(t)$ in the received signal $r(t)$, is a random variable.

The input data is converted into bits, reshaped and modulated with Binary Phase Shift Keying (BPSK) signaling scheme. Square-root raised cosine filter is used to reduce the spectral occupancy and converts the modulated digital signal to analog signal suitable for transmission over the radio channel.

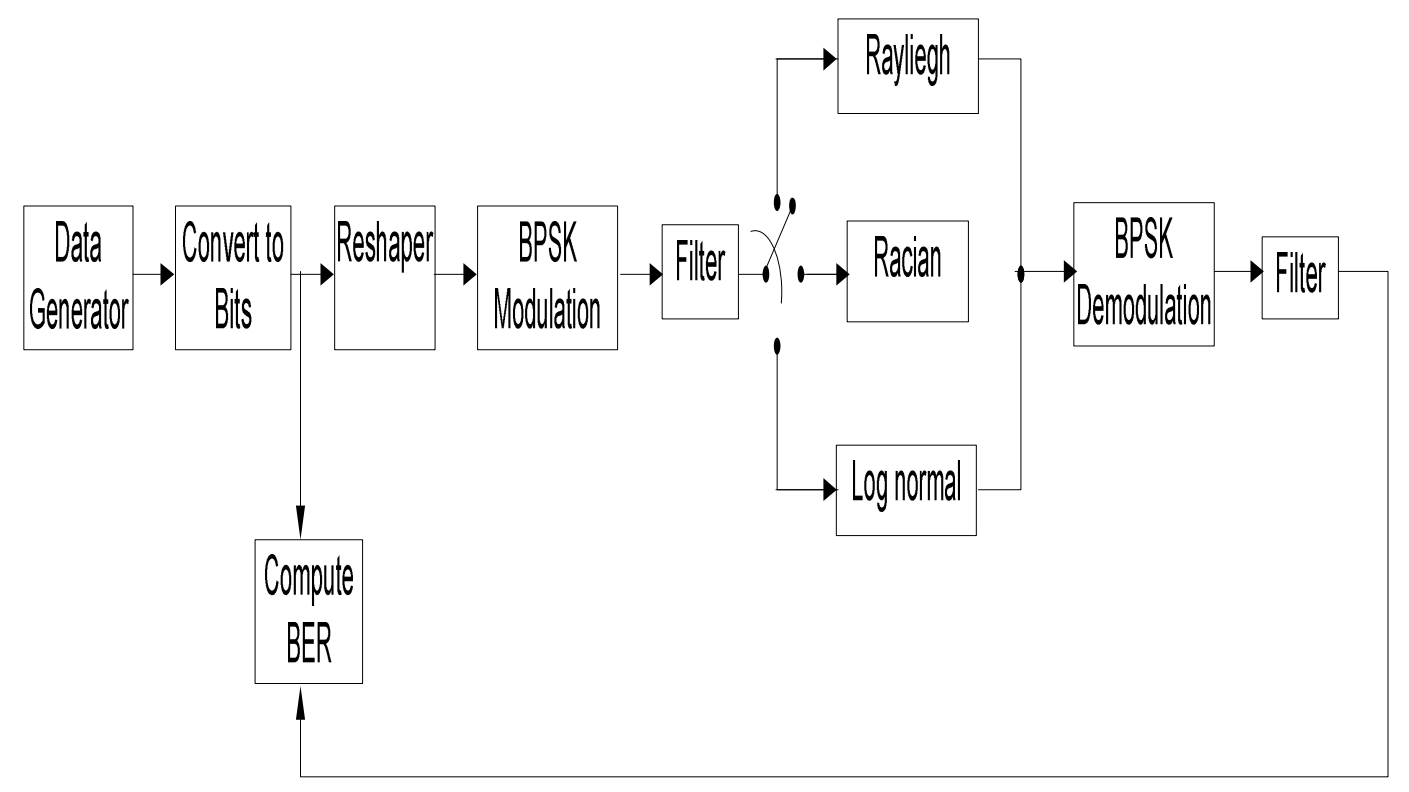

Figure 1. Fading channel selection process

\subsection{Binary Phase Shift Keying (BPSK) Modulation Scheme}

BPSK modulation which is a two level modulation is used for the investigation. In this paper, the phase of the carrier varies in accordance with the message signal. That is the phase of constant carrier amplitude is switched between two values according to the two possible signals corresponding to binary 1 and 0 . The two phases are separated by $180^{\circ}$ [21]. 
International Journal of Wireless \& Mobile Networks (IJWMN) Vol. 4, No. 6, December 2012

\subsection{Analytical Expression}

The BPSK signal is given as

$$
\begin{array}{lll}
S_{B P S K}(t)=\sqrt{\frac{2 E_{b}}{T_{b}}} \cos 2 \pi f_{c} t+\phi_{c} & 0 \leq t \leq T & \text { (binary 1) } \\
S_{B P S K}(t)=\sqrt{\frac{2 E_{b}}{T_{b}}} \cos 2 \pi f_{c} t+\phi_{c}+\pi & 0 \leq t \leq T & \text { (binary 2) }
\end{array}
$$

$$
\begin{gathered}
\text { where } E_{b}=\text { energy per bit } \\
T_{k}=\text { transmitted symboi } \\
\phi_{c}=\text { the phase }
\end{gathered}
$$

BPSK signal is generally represented as

$$
S_{B P S K}(t)=m(t) \sqrt{\frac{2 E_{b}}{T_{b}}} \cos 2 \pi f_{c} t+\phi_{c}
$$

where $m(t)$ is binary data which takes on one of two possible pulse shapes.

\subsection{Doppler Frequency Shift}

Doppler shift is the random changes that occur in a channel introduced as a result of a mobile user's mobility or movement. It is the apparent difference in frequency of the received signals from that of the transmitted signals when there is a relative motion between the transmitter and receiver.

This Doppler frequency shift, $f_{d}$, is given by [14] as:

$$
f_{d}=\frac{v}{c} \cos \theta
$$

where $\theta$ is the angle formed between the incident electromagnetic wave and the moving receiver

$v$ is the mobile speed

$\lambda$ is the wavelength of carrier.

$c$ is speed of light.

\subsection{Rayleigh Distribution}

This is one of the distributions encountered in multipath propagation, this occurs when the envelope of the received signal follows a Rayleigh distribution. It is used to model locations that are heavily shadowed by surrounding buildings as Rayleigh Distribution. Rayleigh distribution is statistically used to model a faded signal when there is no dominant path.

The envelope of the received signal with Rayleigh distribution has the probability density function (pdf) given by [7] as

$$
p(r)=\frac{r}{\sigma^{2}} \exp \left(-\frac{r^{2}}{2 \sigma^{2}}\right) \quad 0 \leq t \leq \infty
$$

where $r$ is the received signal envelope.

$$
\frac{r^{2}}{2} \text { is the instantaneous power. }
$$

$\sigma$ is the root mean square (r.m.s) value of the received signal 


$$
\begin{aligned}
& \sigma^{2} \text { is the local average power of the received signal before envelope } \\
& \text { detection. }
\end{aligned}
$$

\subsection{Rician Distribution}

The Rician distribution which also occurs as a result of multipath propagation is statistically used to model a distribution when a strong line of sight component is present along with the weaker components.

It has the probability density function (pdf) given by [15] as:

$$
p(r / s, \sigma)=\frac{r}{\sigma^{2}} \exp \left(-\frac{r^{2}+s^{2}}{2 \sigma^{2}}\right) I_{0}\left(\frac{s r}{\sigma^{2}}\right) \quad \text { for } s \geq 0, \quad r \geq 0
$$

where $s$ is the peak amplitude of the dominant signal

$$
\begin{aligned}
& I_{o}(\ldots) \text { is zero order Bessel function of the first kind } \\
& \frac{r^{2}}{2} \text { is the instantaneous power } \\
& \sigma \text { is the standard deviation of the local power. }
\end{aligned}
$$

Rician distribution is often described in terms of a parameter, $k$, known as the Rician factor and is expressed by [7] as:

$$
k=10 \log \frac{s^{2}}{2 \sigma^{2}}
$$

As $s$ approaches $0, k$ approaches $\infty \mathrm{dB}$ and as the dominant path decreases in amplitude, the Rician distribution degenerates to a Rayleigh distribution.

\subsection{Log-normal Distribution}

Log-normal distribution describes the random shadowing effects as a result of buildings and other objects on the propagation path which occurs over a large number of measurement locations which have the same transmitter and receiver separation. Therefore, random fluctuations in the mean signal power occur over large distance.

The probability density function (pdf) of a log-normal distribution given by [22] is:

$$
p(s)=\frac{1}{\sigma_{s} \sqrt{2 \pi}} \exp \left(-\frac{\left(S-s_{m}\right)^{2}}{2 \sigma_{s}^{2}}\right)
$$

where $s_{m}$ is the mean value of $\mathrm{S}$ in $\mathrm{dBm}$

$\sigma_{s}$ is the standard deviation of $\mathrm{S}$ in $\mathrm{dB}$

$S$ is the value of $10 \log (\mathrm{s})$ in $\mathrm{dBm}$

$\mathrm{s}$ is the signal power in $\mathrm{mW}$

\subsection{System Performance Measure}

\subsubsection{Bit error rate (BER)}

BER is a performance measure that specifies the number of bits destroyed as they are propagating through the distributions. In this paper, transmission speed and the distribution of the channel affect the BER performance. BER for each of the distributions is determined with equation (11). 


$$
\mathrm{BER}=\frac{\text { number of bits in wror }}{\text { tatal number of bits sent }}
$$

The BER is given by [8] as:

$$
\mathrm{BER}=\int_{0}^{\infty} P_{b}\left(\frac{E}{r}\right) P(r) d r
$$

where $P_{b}\left(\frac{E}{r}\right)$ is the conditional error probability which depends on the BPSK modulation.

$\mathrm{P}(\mathrm{r})$ is the pdf of each of the Rayleigh, Rician, and Log-normal distributions.

\subsection{Transmit and Receive Filter}

In this paper, an identical square root raised cosine pulse shaping filter is used as both the transmit and receive filters so that the input signal can fit in the analog band limited channel to have zero intersymbol interference (ISI) at the pulse sampling time and to reduce the noise power outside of signal bandwidth at the receiver front end [23]. Filtering is achieved by making sure that the overall channel transfer function has a Nyguist frequency response which is the characteristic of square root raised cosine pulse shaping filter in mobile communication [8]. The impulse response of the filter $q_{r c}(t)$ is given by [8] as

$$
q_{r c}(t)=\left\{\frac{\sin \left(\frac{\pi t}{T_{s}}\right)}{\frac{\pi t}{T_{s}}} * \frac{\cos \left(\frac{\pi \beta t}{T_{s}}\right)}{1-\left(\frac{4 \beta t}{2 T_{s}}\right)^{2}}\right.
$$

The transfer function of a raised cosine filter $P_{r c}$ is obtained by taking the Fourier transform of the impulse response which is given by [8] as

$$
P_{r c}=\left\{\frac{T_{s}}{2}\left[1+\cos \left(\frac{\pi\left(|f| \cdot 2 T_{s}-1+\beta\right)}{2 \beta}\right)\right]\right.
$$

$$
\begin{gathered}
0 \leq|f| \leq \frac{(1-\beta)}{2 T_{s}} \\
\frac{(1-\beta)}{T_{s}} \leq|f| \leq \frac{(1+\beta)}{2 T_{s}} \\
|f|>\frac{(1+\beta)}{2 T_{s}}
\end{gathered}
$$

where $\beta$ is the roll off factor which ranges between 0 and 1

$T_{s}$ is the symbol time

Since the spectrum is zero for $|f|>\frac{(1+\beta)}{2 T_{s}}$, the bandwidth BW of the baseband pulse is $\frac{(1+\beta)}{2 T_{s}}$. As $\beta$ increases, the bandwidth of the filter increases and the time side lobe levels decrease in adjacent symbol slots. 
International Journal of Wireless \& Mobile Networks (IJWMN) Vol. 4, No. 6, December 2012

\section{SIMULATION PARAMETERS}

The investigation of the performance of the BPSK signals in each of the distributions previously mentioned is carried out using MATLAB communication and signal processing Toolboxes. The parameters that closely describe the fading channels are used. These parameters are contained in Table 1.

\subsection{Acquisition of Data}

The simulation process was carried out with Random data source which was converted to binary using MATLAB's decimal to binary function, reshaped and modulated with BPSK scheme for transmission to each of the fading distributions. The following parameters were used;

Table1. Simulation Parameters

\begin{tabular}{|l|l|}
\hline Parameters & Variables \\
\hline Length of message, numSymb & 10000 \\
\hline Number of samples per symbols, nSamp & 8 \\
\hline Modulation level, M & 2 \\
\hline Carrier frequency of $900 \mathrm{MHz}, f_{c}$ & $900 \mathrm{e} 6$ \\
\hline Speed of light, c & $3 \mathrm{e} 8$ \\
\hline Mobile speed in m/s, vel.(30, 60, 90 km/h) & $\left(\mathrm{vel}^{*} 1 \mathrm{e} 3\right) / 3600$ \\
\hline Doppler spread, $f_{d}$ & $\left(2 * \mathrm{pi}^{*} f_{c} * \mathrm{vel}.\right) / \mathrm{c}$ \\
\hline Signal-to-noise ratio & $0: 2: 14$ \\
\hline Delay spread & $0.1 e^{-4}-0.1 e^{-4}$ \\
\hline
\end{tabular}

\section{RESULTS AND DISCUSSION}

The BER performance obtained from the BPSK transmission over the three different fading scenarios previously mentioned at the three different mobile speeds are presented in Figures 2, 3 , and 4 . These results are obtained by computer based simulations of the developed algorithms using MATLAB 7.7 application package. Figure 2 shows the simulated BER performance for Rayleigh, Rician and Log-normal distributions using BPSK modulation scheme at mobile speed of $30 \mathrm{~km} / \mathrm{hr}$. It is evident from the result that as the signal to noise ratio (SNR) increase, the BER values for the distributions also decrease. For example at SNR of $4 \mathrm{~dB}$, the BER value over AWGN is 0.0291, Rayleigh is 0.16533, and Rician is 0.090917 while Log-normal is 0.033597. When the SNR is increased to $8 \mathrm{~dB}$, the BER value for AWGN is 0.00019 , Rayleigh is 0.072 , Rician is 0.046917 and Log-normal is 0.010482 . Also at SNR of $14 \mathrm{~dB}$, the values for AWGN, Rayleigh, Rician and Log-normal fading channels obtained are $0,0.0075,0.00625$, and 0.00069669 respectively. From the Figure 2, it can be deduced that the Log-normal fading environment has the lowest BER value with the BPSK modulation technique at uniform speed of $30 \mathrm{~km} / \mathrm{hr}$. Figure 3 also shows the result obtained at mobile speed of $60 \mathrm{~km} / \mathrm{h}$ for the three scenarios. It is evident from the result that as the SNR values are increasing, the BER values for the distributions are also decreasing. For example at SNR of $2 \mathrm{~dB}$, the BER value for AWGN channel is 0.00444 , Rayleigh is 0.4045 , and Rician is 0.16533 while Log-Normal is 0.033597 . When the SNR is increased to $6 \mathrm{~dB}$, the BER value for AWGN channel is 0.0012 , Rayleigh is 0.23933 , Rician is 0.146 and Log-normal is 0.042001 . It is observed that at mobile speed of $60 \mathrm{~km} / \mathrm{hr}$, for SNR value considered BER values of the various fading environments are higher than at the mobile speed of $30 \mathrm{~km} / \mathrm{h}$. Also, log-normal environment gives the lowest BER value. 
International Journal of Wireless \& Mobile Networks (IJWMN) Vol. 4, No. 6, December 2012

The result obtained in Figure 4 at $90 \mathrm{~km} / \mathrm{h}$ for the three distributions follow the same trend except the BER values are higher at all SNR. At higher SNR, which is from $14 \mathrm{~dB}$, the performance obtained through the Rayleigh and Rician are almost the same. This is because high power will be transmitted, and as a result it will be very difficult to experience the effect of dominant component in the Rician distribution. The results obtained are justifiable in that the effect Doppler spread is noticeable at high speeds and affect the BPSK transmitted signals. Also, where there is no line of sight, it means that many obstacles are along the path hence high error will occur as expected. Also since the interference levels present in a communication system are generally set by external factors due to those distributions and cannot be changed by the system design, then the bandwidth of the system are kept constant with the filter used to reduce the error to be achieved by reducing the bandwidth with the constant power level. Though, each type of modulations performs differently in the presence of different fading, therefore, BPSK signalling scheme reduces the effect of fading more than higher order of PSK modulation scheme. The highest BER values observed in Rayleigh fading indicates the highest number of error received out of 1000 bit transmitted and worst performance of the system while log-normal which gives the least bit errors received indicates the best performance with the system. The performance of thr Rician is in between. To select the best fading model in the communication design requires selecting the one which gives the highest bit errors because if the wireless communication system can perform very well in this worst model, it will perform excellently in other channels. The results obtained in this paper is in agreement with the work of [15] where many experimentally and theoretically based models have been proposed to quantitatively evaluate and combat the fading phenomenon. In his work, minimum description length (MDL) criterion for model selection is proposed and the measurements were taken in a sub-urban environment.

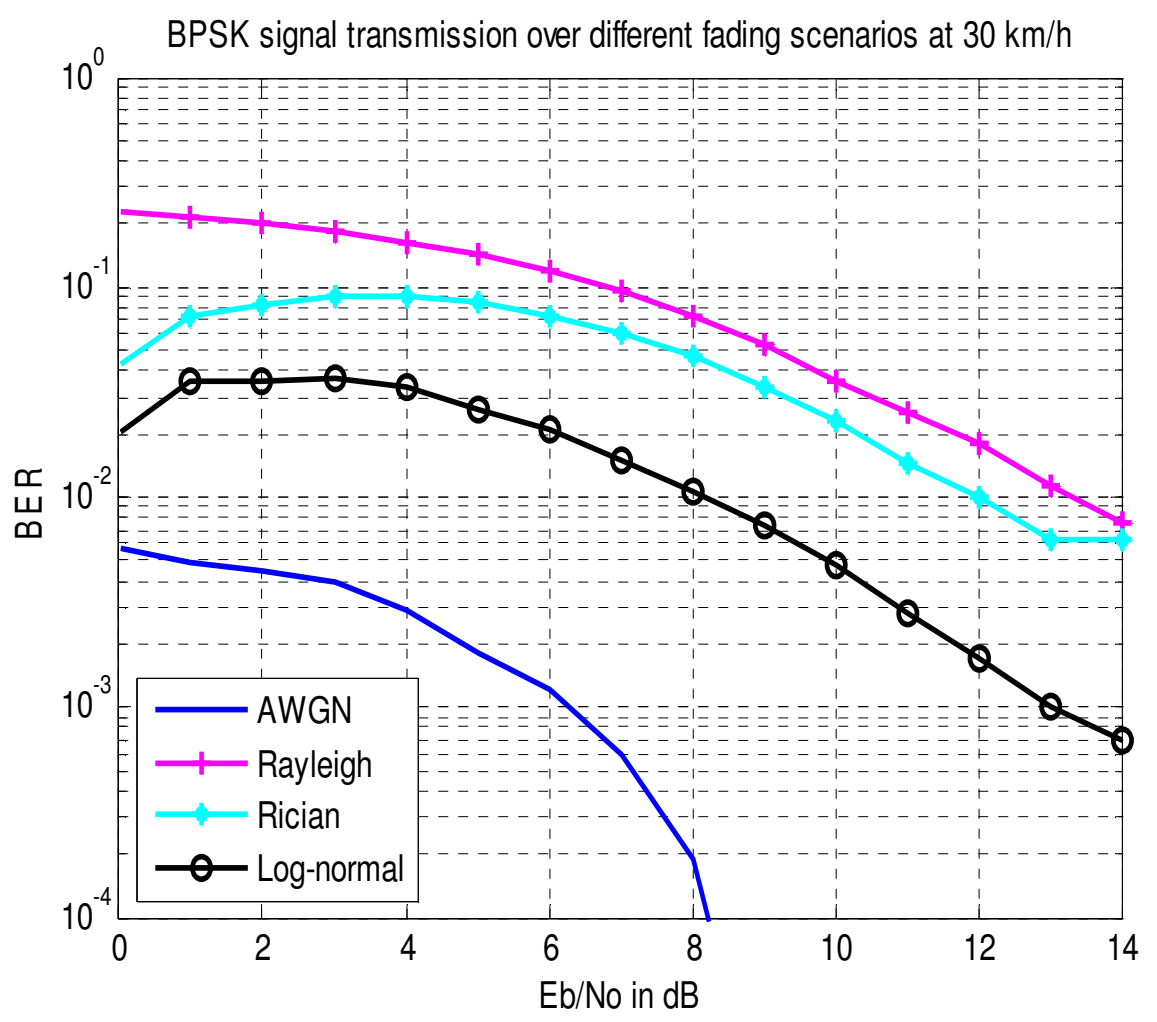

Figure 2. Simulation of BER versus SNR over Rayleigh,

Rician, and log-normal distributions at $30 \mathrm{~km} / \mathrm{h}$. 
International Journal of Wireless \& Mobile Networks (IJWMN) Vol. 4, No. 6, December 2012

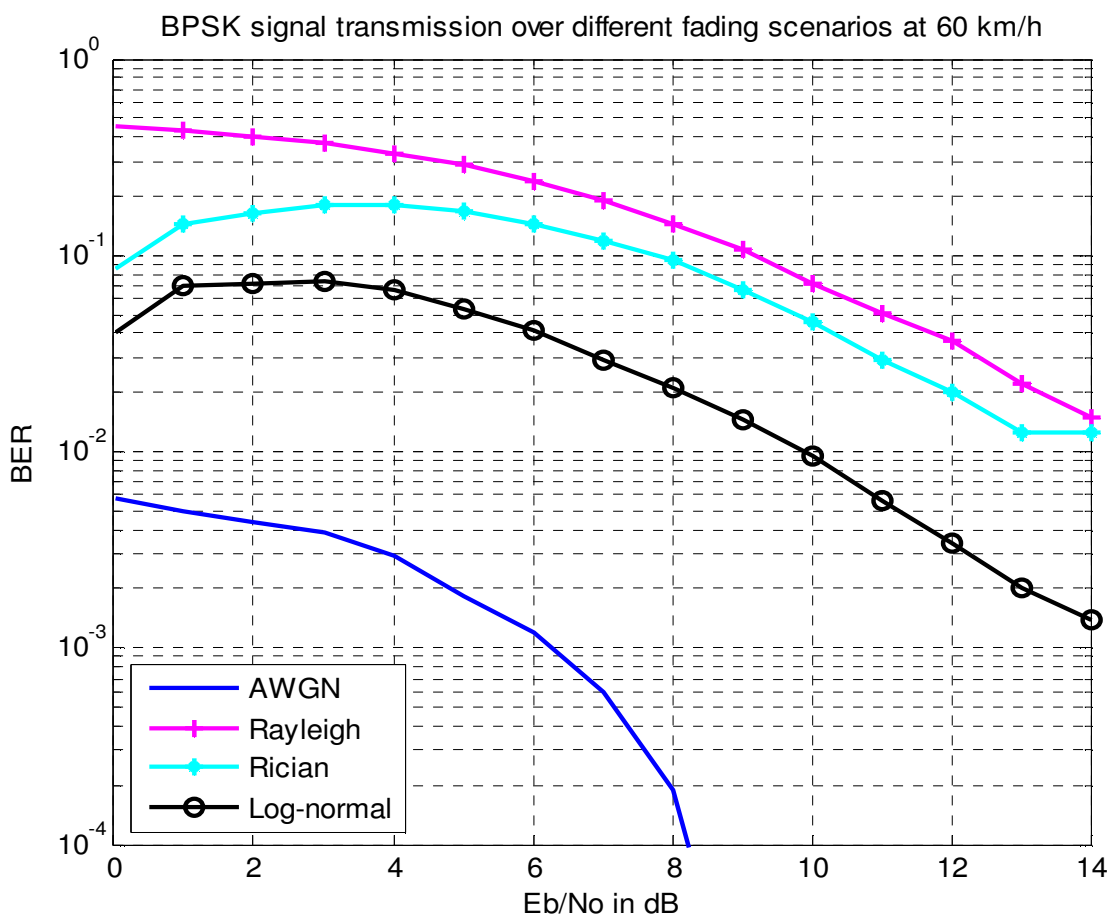

Figure 3. Simulation of BER versus SNR Rayleigh, Rician and $\log$-normal distributions at $60 \mathrm{~km} / \mathrm{h}$.

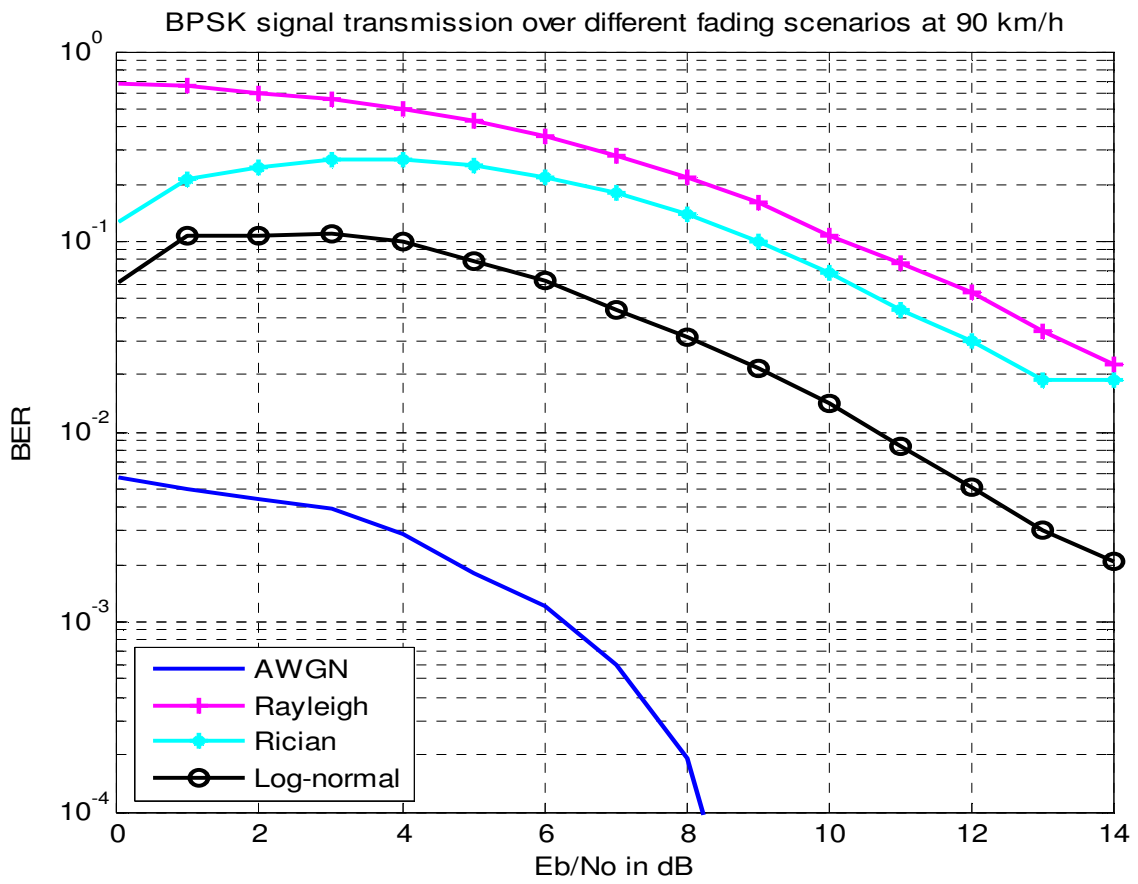

Figure 4. Simulation of BER versus SNR over Rayleigh, Rician and $\log$-normal distributions at $90 \mathrm{~km} / \mathrm{hr}$. 


\section{CONCLUSIONS}

In this paper, the performance of fading channel models for mobile wireless transmission system using BPSK signal with MATLAB communication Toolbox has been evaluated in term of BER. The system model for the received signal has been obtained in the presence of the Rayleigh, Rician, and log-normal distributions formed from multipath propagation and BPSK signals, the noise is modeled as the ideal (AWGN) channel. The AWGN channel served as a benchmark to determine the number of iterations to be used for the process in the sub-urban environment. BER has been obtained for each of the distributions using the probability density function of the fading distributions and the conditional error probability which depends on BPSK signal. The BER results obtained at mobile speed of $30 \mathrm{~km} / \mathrm{hr}$ with the SNR of $2 \mathrm{~dB}$ measured at the same point for AWGN, Rayleigh, Rician and Log-normal fading are 0.00444, $0.20225,0.082667$ and 0.036196 indicating $0 \%, 20 \%, 8 \%$ and $3.2 \%$ bits error respectively. Also at an increased mobile speed of $60 \mathrm{~km} / \mathrm{hr}$ with the same power, the BER values obtained for AWGN, Rayleigh, Rician and Log-normal are 0.00444, 0.4045, 0.16533 and 0.072392 indicating $0 \%, 40 \%, 16.6 \%$, and $7 \%$ bits error respectively while mobile speed of $90 \mathrm{~km} / \mathrm{hr}$ gives BER values of $0 \%, 60 \%, 24.8 \%$ and $10.8 \%$ respectively. The comparisons are made from the results obtained to determine the best out of the three different fading channels. It has been confirmed that Log-normal fading channel gives the lowest BER value while Rayleigh gives the highest bits. Based on the results obtained in this paper, Rayleigh distribution which gives the highest BER is the best distribution to be used by mobile wireless communication engineer in the design of robust system before the implementation because if the system can perform very well in Rayleigh distribution it will perform better in other distributions.

\section{REFERENCES}

[1] Iskander, C.D (2011), “A MATLAB Based Object-oriented Approach to Multipath Fading Channel Simulation” Hi-Tek Multisystems White paper, Quebec, Canada.

[2] Bernard, S. (1997), Rayleigh Fading Channels in Mobile Digital Communication System, IEEE communication magazine, vol. 35 , no. 7 pp.90-100.

[3] Adeyemo, Z.K. and Raji, T.I. (2010), Bit Error Rate Analysis for Wireless Links Using Adaptive Combining Diversity, Journal of Theoretical and Applied Information Technology, vol. 20, no.8,pp. 58-66.

[4] Mohamed-slim., Tang and Goldsmith (1999), An Adaptive Modulation Scheme for Simultaneous Voice and Data Transmission over Fading Channels, IEEE Journal on Selected Areas in Communications, vol.17, no.5, pp.837-847.

[5] Xuanming, D. (2006), Effect of Slow Fading and Adaptive Modulation on TCP (UDP) Performance of High-speed packet Wireless Networks, Technical Report no UCB/EECS-2006109, University of California, Berkeley.

[6] Patzold, M. (2002), Mobile Fading Channels, $1^{\text {st }}$ Edition, John Wiley and Sons, Norway.

[7] Vijay G. K. (2007), Wireless Communications and Networking. Morgan Kaufmann, United States of America.

[8] Rappaport, T. S. (2002), Wireless Communications: Principles and Practice $2^{\text {nd }}$ Edition, Prentice Hall.

[9] Rappaport, T. S. (1989), Characterization of UHF Multipath Radio Channels in Factory Buildings, IEEE Transaction on Antennas and Propagation, vol.37, no.8, pp.1058-1069.

[10] Steven, W., Jeffrey, G. A. and Nihar, J. (2007), The Effect of Fading, Channel Inversion, and Threshold Scheduling on Ad Hoc Networks, IEEE Transmissions on Information Theory, vol.53, no.11 pp.4127-4149. 
International Journal of Wireless \& Mobile Networks (IJWMN) Vol. 4, No. 6, December 2012

[11] Parastoo, S. and Predrag, R. (2008), On Information Rate of Time-varying Fading Channels Modeled as Finite-State Markov Channels, IEEE Transactions on Communication, vol.56, no.8, pp.1268-1278.

[12] Ramjee, P. and Hiroshima, H. (2002), Simulation and Software Radio for Mobile Communications, Communication Research Laboratory, Independent Administrative Institution, Japan.

[13] Gregory, D. D., Theodore, S. R., and David, A. W (2002), New Analytical Models and Probability Density Functions for Fading in Wireless Communications, IEEE Transactions on Communications, vol.50, no.6, pp.1005-1012.

[14] Hyunseung, C. (2006), Mobile Radio Propagation/Channel Coding Mobile Computing. Sungkyunkwan University retrieved March 11, 2011, from (www.google.com).

[15] Taneda M.A., Takada J., and Araki K. (2001), The Problem of the Fading Model Selection. IEICE Transaction on Communication, vol. E84 B, no. 3, pp 660-666.

[16] Peppas, K.P., Nistazakis and Tumbras, G.S. (2011), "An Overview of the Physical insight and the various Performance Metrics of Fading Channels in Wireless Communication Systems" Advanced Trends in Wireless Communications. Retrieved from http://www.intechopen.com/

[17] Zhang, Y. and Fujise, M. (2006), "performance Analysis of Wireless Networks over Rayleigh Fading Channel", IEEE Transactions on Vehicular Technology, vol. 55, no. 5, pp. 1621-1632.

[18] Charalambons, C.D, Buttitude, R.J.C., Li, X. and Zhan, J. (2008), "Modeling Wireless Fading Channels via Stochastic Differential Equations, Identification and Estimation Based in Noisy Measurements" IEEE Transaction on Wireless Communications, vol. 7,no. 2, pp. 434-439.

[19] Tellambura, C. (2008), "Bound on the Distribution of a Sum of Correlated log-normal Random Variables and their Applications" IEEE Transaction on Communications, vol. 56, no. 8, pp.1241-1248.

[20] Young, J.C. and Saewong, B., (2008), "Multichannel Wireless Scheduling under Limited Terminal Capacity" IEEE Transactions on Wireless Communications, vol. 7, no. 2, pp. 611-617.

[21] Rashmi, S., Sunil, J., and Navneet, A. (2011), Performance Analysis of Different M-ary Modulation Techniques in Cellular Mobile Communication, In Multimedia Communications, A special Issue from IJCA, retrieved on $22^{\text {nd }}$ may, 2011 from (www.ijcaonline.org).

[22] Log-normal distribution (online) retrieved May 21, 2011, from (www.wikipedia.com/).

[23] Shuwei, H. (2002),"Evaluation Techniques for TDMA Cellular Radio with Co-channel Interference". Unpublished PhD Thesis, University of Calgary, Alberta.

\section{Authors}

Zachaeus K. Adeyemo received the B.Eng. and M.Eng. degrees in Electrical Engineering from University of Ilorin, Ilorin, Nigeria and his Ph.D. degree in Electronic and Electrical Engineering from Ladoke Akintola University of Technology (LAUTECH), Ogbomoso, Nigeria. He is a member of the IEEE and a registered member of Council for the Regulation of Engineering in Nigeria (COREN). His research interest is on signal processing in mobile communications.

Damilare O. Akande received his B.Tech (Electronic and Electrical Engineering) and M.Eng (Communication) in 2008 and 2012 respectively. He is currently a lecturer at Ladoke Akintola University of Technology, Ogbomoso. His field of study are antenna design and wireless communications

Festus K. Ojo received his B.Tech (Electronic and Electrical Engineering) and M.Eng (Communication) in 2008 and 2012 respectively. He is currently a lecturer at Ladoke Akintola University of Technology, Ogbomoso. His field of study is wireless communications 
International Journal of Wireless \& Mobile Networks (IJWMN) Vol. 4, No. 6, December 2012

Hamdat O. Raji received his B.Tech (Electronic and Electrical Engineering) in 2011. She intends pursuing her M.Tech in Wireless communication. 